\title{
Universiteit
}

Leiden

The Netherlands

\section{Large-scale finite-wavelength modulation within turbulent shear flows}

Prigent, A.; Grégoire, G.; Chaté, H.; Dauchot, O.; Saarloos, W. van

\section{Citation}

Prigent, A., Grégoire, G., Chaté, H., Dauchot, O., \& Saarloos, W. van. (2002). Large-scale finite-wavelength modulation within turbulent shear flows. Physical Review Letters, 89(1), 014501. doi:10.1103/PhysRevLett.89.014501

Version: $\quad$ Not Applicable (or Unknown)

License: $\quad$ Leiden University Non-exclusive license

Downloaded from: https://hdl.handle.net/1887/66589

Note: To cite this publication please use the final published version (if applicable). 


\title{
Large-Scale Finite-Wavelength Modulation within Turbulent Shear Flows
}

\author{
Arnaud Prigent, ${ }^{1}$ Guillaume Grégoire, ${ }^{1}$ Hugues Chaté, ${ }^{1}$ Olivier Dauchot, ${ }^{1}$ and Wim van Saarloos ${ }^{2}$ \\ ${ }^{1}$ CEA-Service de Physique de l'État Condensé, Centre d'Études de Saclay, 91191 Gif-sur-Yvette, France \\ ${ }^{2}$ Instituut-Lorentz, Universiteit Leiden, Postbus 9506, 2300 RA Leiden, The Netherlands
}

(Received 2 October 2001; published 14 June 2002)

\begin{abstract}
We show that turbulent "spirals" and "spots" observed in Taylor-Couette and plane Couette flow correspond to a turbulence-intensity modulated finite-wavelength pattern which in every respect fits the phenomenology of coupled noisy Ginzburg-Landau (amplitude) equations with noise. This suggests the existence of a long-wavelength instability of the homogeneous turbulence regime.
\end{abstract}

DOI: $10.1103 /$ PhysRevLett.89.014501

One of the fascinating phenomena in fluid mechanics and pattern formation is the coexistence in space and time of regions where the flow is disordered with others where it is laminar. Already noted by Feynman as an outstanding problem [1], the origin and structure of these turbulent patches (spots, slugs, etc.) commonly observed in shear flows at moderate Reynolds numbers $R$ [2-4] remains largely unknown. Particularly striking is the so-called "spiral" or "barber pole turbulence" regime of the TaylorCouette flow (TCF), in which typically one helical stripe of disordered fluid motion appears in the otherwise laminar flow between two counterrotating cylinders [5-7]. Similarly, while plane Couette flow (PCF) between two plates is linearly stable at all $R$, it exhibits localized turbulent spots of intriguing dynamical nature [8-10]. In many systems, the first appearance of patterns is associated with an instability of a homogeneous, essentially noiseless state to a spatially periodic state; the formation and dynamics of such deterministic patterns just above onset can be described in terms of so-called amplitude or GinzburgLandau equations.

In this Letter, we report a remarkable empirical observation: in our large aspect ratio experiments on both $\mathrm{PCF}$ and TCF, localized turbulent patches actually appear as the result of a well-defined transition to a finite-wavelength turbulent pattern, which in every respect fits the phenomenology of amplitude equations with noise. Since the patterns correspond to a spatial modulation of the turbulence intensity, our findings yield empirical evidence that homogeneous turbulence in PCF and TCF can exhibit an "instability" to a modulated state which, at the phenomenological level, is the noisy analog of the primary bifurcation in noiseless pattern-forming systems.

Our PCF and TCF experimental setups have already been described elsewhere $[8,9,11]$. The PCF results have been obtained with a gap $d=1.5 \mathrm{~mm}$ and aspect ratios $\Gamma_{x}=385$ along the flow direction and $\Gamma_{z}=170$ perpendicular to it. The TCF cylinders (inner and outer radii $r_{\mathrm{i}}=49.09 \mathrm{~mm}$ and $\left.r_{\mathrm{o}}=49.96 \mathrm{~mm}\right)$ are separated by a gap $d=0.872 \mathrm{~mm}$ giving azimuthal and axial aspect ratios $\Gamma_{\theta}=357$ and $\Gamma_{z}=430$. In both experiments, optical techniques allow for the visualization of the whole
PACS numbers: 47.20.Ft, 47.27.Cn, 47.54.+r, 83.60.Wc

flow seeded with Kalliroscope flakes. In the following, the Reynolds number is defined as $R=U d / \nu$ where $\nu$ is the kinematic velocity of the fluid (water + Kalliroscope). For the PCF, $\pm U$ is the velocity of the planes, while for the TCF the "inner" and "outer" Reynolds numbers $R_{\mathrm{i}, \mathrm{o}}$ are based on $U=r_{\mathrm{i}, \mathrm{o}} \Omega_{\mathrm{i}, \mathrm{o}}$ with $\Omega_{\mathrm{i}, \mathrm{o}}$ as the inner/outer angular velocity.

In Figs. 1a and $1 \mathrm{~b}$ we show typical snapshots of the flow patterns of interest here: oblique stripes form a fairly regular pattern with wavelengths of the order of $40-60 \mathrm{~d}$. The largeness of this scale reveals why only a single "turbulent spiral" could be observed in previous TCF experiments [5,6]. Similarly, previous PCF studies could produce only one or two turbulent "spots" taking sometimes the shape of an inclined stripe [8,9]. Laser Doppler velocimetry (LDV) measurements in the TCF case show that both the local velocity and its fluctuations - which can be taken as a measure of the turbulent intensity - are modulated with the same wavelength as the light intensity (Fig. 1c). The lighter regions are more turbulent. Moreover, the amplitude of the modulations of the velocity fluctuations and of the light intensity are proportional as the Reynolds number is varied (Figs. 1d). The spiral turbulence regime thus corresponds to a large-but-finite wavelength modulation of the strength of turbulence. In our experiments, the regular pattern was observed for $680<R<830$ in the PCF, and in a fairly large region of the $\left(R_{\mathrm{i}}, R_{\mathrm{O}}\right)$ parameter plane in our TCF apparatus (Fig. 2a).

The similarity between the PCF and TCF patterns goes beyond the above qualitative description. We first note that, in the TCF case, the stripe pattern rotates in the laboratory frame with the mean angular velocity $\frac{1}{2}\left(\Omega_{\mathrm{i}}+\right.$ $\Omega_{\mathrm{o}}$ ). When both the TCF and PCF patterns are stationary (i.e., when $\Omega_{\mathrm{i}}=-\Omega_{\mathrm{o}}$ for the TCF), they exhibit the same wavelengths (in units of $d$ ) at any Reynolds number (Fig. 2b).

As the PCF experiment is rather difficult to control for small gaps, we hereafter focus on our TCF data; nevertheless, all our observations suggest that the essential findings are common to both experiments.

In most of the relevant region of the $\left(R_{\mathrm{i}}, R_{\mathrm{O}}\right)$ parameter space, the azimuthal wave number is quantized by the 
(a)

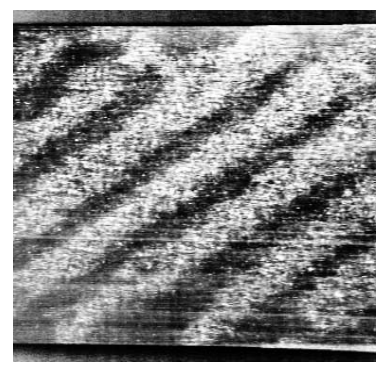

(c)

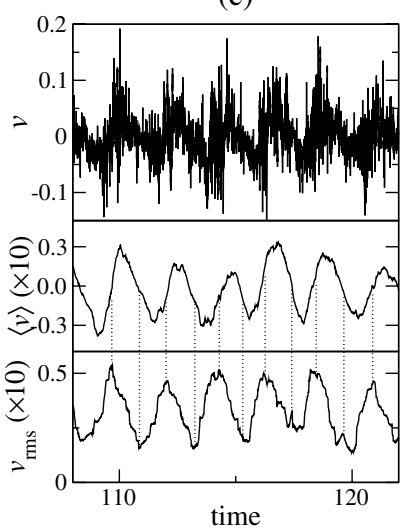

(b)

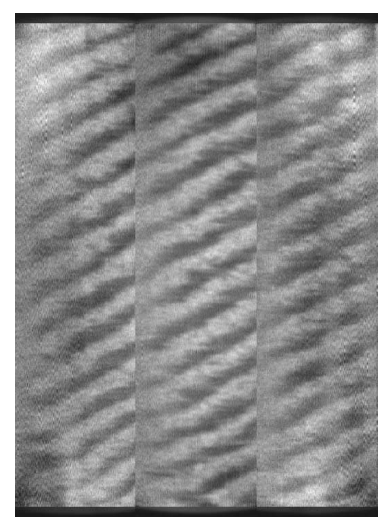

(d)

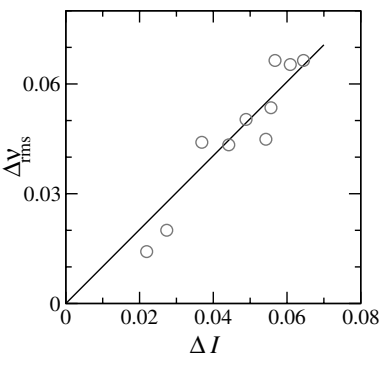

FIG. 1. Snapshots of the PCF [(a) $R=716]$ and TCF [(b) $\left.R_{\mathrm{i}}=703, R_{\mathrm{o}}=-699\right]$. The flow is in the horizontal direction in both cases. (b) The two vertical lines are due to the optical system. (c) Local axial velocity time series $v(t)$ (in $\mathrm{m} / \mathrm{s}$, time in s) measured at midgap and midheight with LDV in the TCF for $d=1.85 \mathrm{~mm}, R_{\mathrm{i}}=670$, and $R_{\mathrm{o}}=-850$. Top: raw signal; middle: running average of $v(t)$ over windows of $0.4 \mathrm{~s}$; bottom: rms of previous average. The dotted lines indicate that the fluctuations are smallest where the average velocity increases through zero. (d) $\Delta v_{\text {rms }}$, amplitude of the modulation of the rms of $v(t)$ [calculated as in (c)], vs $\Delta I$, amplitude of the modulation of the light intensity $I$ for various $R_{\mathrm{i}}$ between 640 and 730 [other parameters as in (c)].

system circumference and hence constant: we observe six stripes. In view of this, it is sufficient to record only the light intensity $I$ as a function of the $z$ coordinate along a line parallel to the axis of the cylinder. If we keep $R_{\mathrm{O}}$ fixed and decrease $R_{\mathrm{i}}$ the modulation amplitude of the light intensity and concomitantly the modulation ampli-

(a)

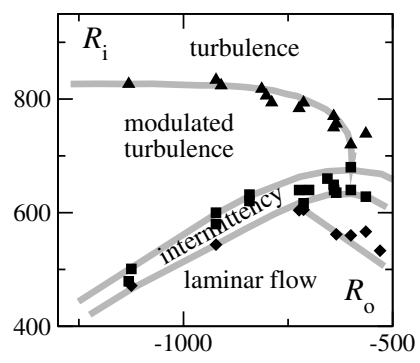

(b)

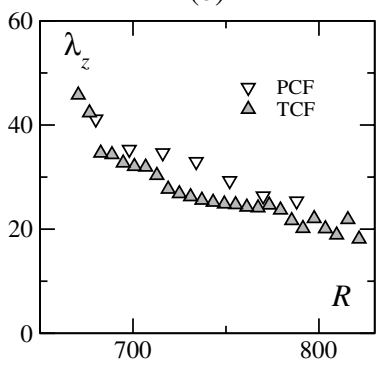

FIG. 2. (a) Phase diagram of our TCF experiment; (b) wavelength of the pattern in the transversal/axial direction as a function of $R$ for PCF and TCF (in units of the gap $d)$. For the TCF, $R_{\mathrm{i}}=-\eta R_{\mathrm{o}}$ and $R=R_{\mathrm{i}} /(1+\eta)$.

tude of the velocity fluctuations grow in a continuous fashion reminiscent of a supercritical bifurcation upon crossing the transition from the fully turbulent regime at the top of Fig. 2a to the intermittent regime (we quantify this below in Fig. 4). Regular patterns such as those shown in Fig. 1 break the $z \rightarrow-z$ parity symmetry. They are often reached after a transient during which " $+z$ " and " $z$ " domains, separated by wandering domain walls or fronts, compete (Fig. 3a). However, near the instability threshold (at high $R_{\mathrm{i}}$ ), domains of both types fluctuate and new ones get spontaneously "nucleated" (Fig. 3b). These observations lead us to analyze the emergence of modulated turbulence stripes within the usual framework of amplitude equations by writing $I(z, t)$ in terms of two slowly varying complex fields $A^{+}$and $A^{-}$[12]:

$$
\begin{aligned}
I(z, t)= & A^{+}(z, t) \exp \left[i\left(k_{0} z-\omega_{0} t\right)\right] \\
& +A^{-}(z, t) \exp \left[i\left(-k_{0} z-\omega_{0} t\right)\right]+\text { c.c. }
\end{aligned}
$$

where $k_{0}$ and $\omega_{0}$ are the basic scales of the pattern. Standard demodulation techniques [13] applied to our experimental data then yield the amplitudes.

We now proceed to a detailed analysis of our $I(z, t)$ TCF data. All results presented below are for an outer Reynolds number $R_{\mathrm{o}}=-1200$ and varying $R_{\mathrm{i}}$. Similar conclusions were reached at different $R_{\mathrm{O}}$ values.

First we record the variation of $\mathcal{A} \equiv\left\langle\left|A^{+}\right|+\left|A^{-}\right|\right\rangle$, the mean modulus of the amplitude(s), with the Reynolds number. Figure 4a shows that $\mathcal{A}^{2} \propto\left(R_{\mathrm{c}}-R_{\mathrm{i}}\right)$ over some range; this is the scaling for the pattern amplitude that one normally finds for a stationary pattern just above the onset of instability, but do note that the linear law here breaks down close to $R_{c}$ : For $R_{\mathrm{i}}$ larger than some value $R_{\text {nucl }} \simeq$ 750, new domains constantly "nucleate" (Fig. 3b). This feature, which inhibits a meaningful measure of the amplitudes, is a direct consequence of the fact that we probe a transition in a strongly fluctuating (turbulent) regime. This motivates us to model the transition to modulated turbulence by the usual coupled amplitude equations that (a)

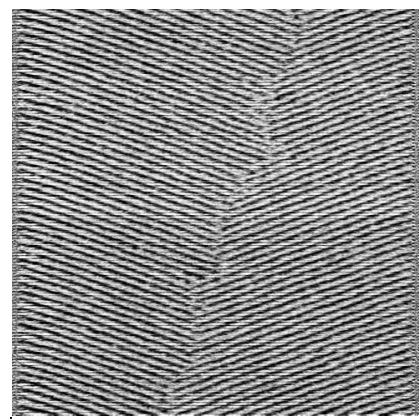

(b)

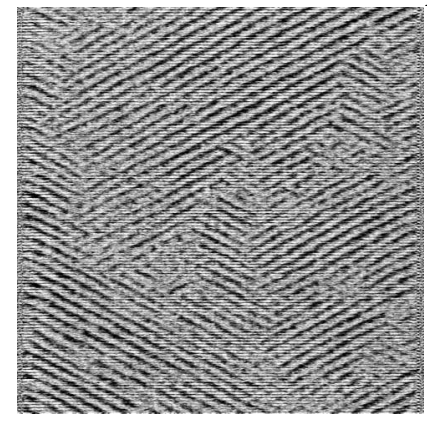

FIG. 3. Space-time diagram of $I(z)$ in the TCF at $R_{\mathrm{O}}=-850$. Time is running upward for $40 \mathrm{~s}$, the full $z$ domain $(37.5 \mathrm{~cm})$ is shown horizontally. (a) $R_{\mathrm{i}}=740$ : transient domain competition. (b) $R_{\mathrm{i}}=775$ : regime with incessant "nucleation" of domains. 
(a)

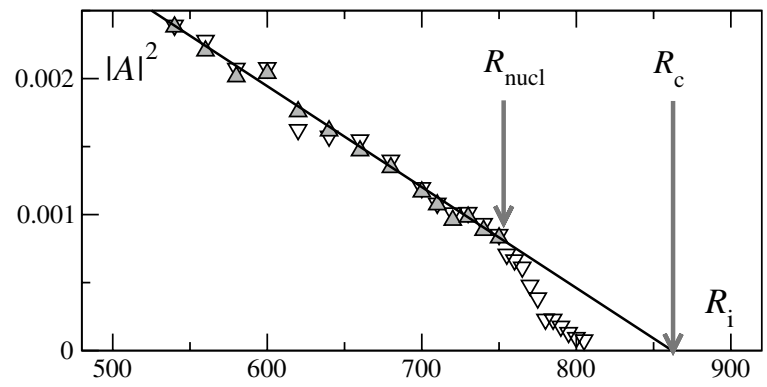

(b)

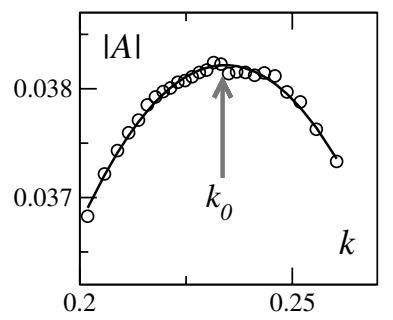

(c)

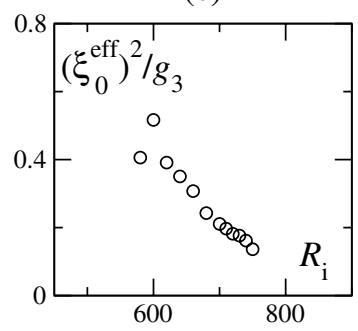

FIG. 4. Analysis of the amplitude $A$ of TCF data at $R_{\mathrm{o}}=$ -1200. (a) Open triangles: $\mathcal{A}^{2}=\langle|A|\rangle^{2}$ vs $R_{\mathrm{i}}$ measured from space-time regions containing various + and - domains. This variation is linear for $R_{\mathrm{i}}$ less than $R_{\text {nucl }}$, the value beyond which spontaneous "nucleation" of + and - domains is observed. Filled triangles: $|A|\left(k_{0}\right)$ vs $R_{\mathrm{i}}$ determined from analysis of monodomain regions. The linear fit yields the critical threshold $R_{\mathrm{c}}=857 \pm 5$. (b) $|A|$ as a function of wave number $k=\partial_{z} \phi$ for $R_{\mathrm{i}}=640$ from the demodulation of data from a monodomain region. The line is a parabolic fit. (c) $\left(\xi_{0}^{\text {eff }}\right)^{2} / g_{3}$ as a function of $R_{\mathrm{i}}$, as extracted from the curvature of parabolic fits like in (b).

described the emergence and dynamics of patterns just above onset in deterministic systems, but with an additive noise term included to model the fluctuations:

$$
\begin{aligned}
\tau_{0} \partial_{t} A^{ \pm}= & {\left[\varepsilon+\xi_{0}^{2} \partial_{z}^{2}-g_{3}\left|A^{ \pm}\right|^{2}-g_{2}\left|A^{\mp}\right|^{2}\right] A^{ \pm} } \\
& +\alpha \eta^{ \pm}(z, t) .
\end{aligned}
$$

Similar amplitude equations with noise have, e.g., been used for liquid crystals [14]. Here $\tau_{0}$ and $\xi_{0}$ are the characteristic time and space scales of the modulations of the amplitudes, $\varepsilon=\left(R_{\mathrm{c}}-R_{\mathrm{i}}\right) / R_{\mathrm{c}}$ is the reduced threshold, $\alpha$ is the noise strength, and $\eta^{ \pm}(z, t)$ is a deltacorrelated white noise. Without noise, the above equations are the standard real Ginzburg-Landau equations for two coupled, symmetric, stationary patterns. The basic + and - stripe patterns are taken to be stationary because extensive analysis of the data on both PCF and TCF [15] shows that they do not move in the frame in which the mean flow vanishes. Apart from parameters like $\tau_{0}$ and $\xi_{0}$ which set the scales, the coupled amplitude equations contain essentially two nontrivial parameters. The ratio $g_{2} / g_{3}$ determines the relative suppression of one type of pattern by the other: if $g_{2} / g_{3}>1$ the dynamics drives the systems to states with domains where only one of the amplitudes is nonvanishing [12]. Our empirical experimental observation that we have either + or - domains therefore requires us to take $g_{2} / g_{3}>1$ [12]. As we shall see, the value 1.2 gives a reasonable and self-consistent description of the dynamical behavior we observe.

The effective noise strength in our simulations of (1) is determined as follows. In the noiseless case $(\alpha=0)$, the amplitudes in (1) always scale as $|A|^{2} \propto \varepsilon$. With noise, the noise-averaged amplitudes in our simulations do exhibit this scaling for large enough $\varepsilon$, as one would expect, but this linear law breaks down near threshold. For small $\varepsilon$, domain "nucleation" fluctuations become important, just as we see in our experiments [Figs. 3b and 4a]. The crossover value $R_{\text {nucl }}$ depends on the noise strength $\alpha$ [16]. We determine $\alpha$ by the requirement that it yields the same $\varepsilon$ value for the onset of spontaneous nucleation of domains as in the experiment. This gives $\alpha \approx 3 \times 10^{-3}$ [17] in dimensionless units obtained by writing $z$ and $t$ in the amplitude equations in units of $\xi_{0}$ and $\tau_{0}$.

We now extract the length and time scales $\xi_{0}$ and $\tau_{0}$ in Eqs. (1) from our experimental data. For deterministic pattern-forming systems, this can be done straightforwardly from the temporal growth of the amplitude and the dependence of $A^{ \pm}(k)$ on wave number $k$ within sufficiently large domains, but for our case this has to be done in parallel with numerical simulations, as the fluctuations renormalize the parameters. Thus the "bare" parameters $\xi_{0}$ and $\tau_{0}$ entering Eq. (1) are renormalized to effective length and time scales $\xi_{0}^{\text {eff }}$ and $\tau_{0}^{\text {eff }}$ which capture the behavior of simulations and experiments in the fitting procedure described below.

For each set of parameter values in the range where no nucleation of opposite-amplitude domains is observed, several (typically four) single-domain space-time regions are selected and the data are demodulated [13]. The variation of the local modulus $|A|$ and phase gradient $\partial_{z} \phi$ with the local wave number $k=\partial_{z} \phi$ is then calculated from all points in each of these space-time domains. The parabolic shape of $|A(k)|^{2}$ (Fig. 4b) is characteristic of Ginzburg-Landau equations such as Eqs. (1): without noise, the amplitude of (near) periodic patterns obeys $\left|A^{ \pm}\right|^{2}=\left[\varepsilon-\xi_{0}^{2}\left(k-k_{0}\right)^{2}\right] / g_{3}$, and we checked numerically that the parabolic shape of $\left\langle|A(k)|^{2}\right\rangle$ is preserved in the noisy equations. Hence a parabolic fit yields estimates of $\varepsilon / g_{3}=\max _{k}|A|^{2}, \xi_{0}^{2} / g_{3}$, and $k_{0}$. We find that coefficient $g_{3}$ is only slightly renormalized by the noise, whereas $\xi_{0}^{\text {eff }}$ can be much smaller than its bare value.

Repeating the above data treatment at various $R_{\mathrm{i}}$ values in the region of interest, we find the expected linear dependence of $\max _{k}|A|^{2}=\varepsilon / g_{3}$ on $R_{\mathrm{i}}$ (Fig. 4a). In fact, this provides another way of determining the threshold $R_{\mathrm{c}}$, as well as a precise estimate of the (effective) nonlinear coefficient $g_{3}=156 \pm 20$. The same series of data also reveals a variation of $\left(\xi_{0}^{\text {eff }}\right)^{2} / g_{3}$ with $\varepsilon$ (Fig. 4 c). As $g_{3}$ is constant and not significantly renormalized by the noise, it is the effective coherence length $\xi_{0}^{\text {eff }}$ which decreases with $\varepsilon$. Numerical simulations of Eqs. (1) reveal that in the presence of noise the effective coherence length $\xi_{0}^{\text {eff }}$ does vary precisely like in the experiments (Fig. 5a). This 
(a)
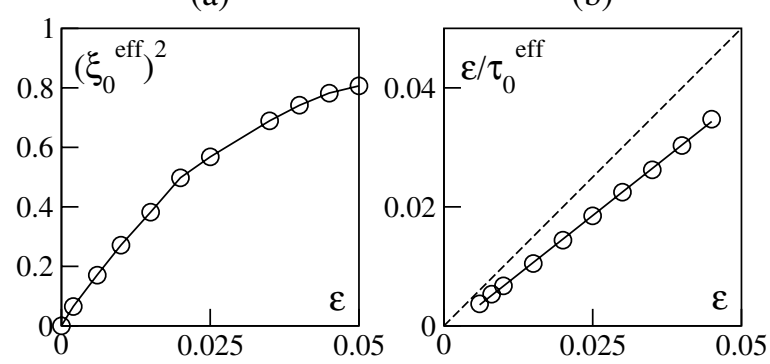

FIG. 5. Numerical simulations of Eqs. (1), varying $\varepsilon$ for $g_{2} / g_{3}=1.2$, and $\alpha=0.003$. (a) Effective squared coherence length $\left(\xi_{0}^{\text {eff }}\right)^{2}$ as a function of $\varepsilon$. Note the similarity with Fig. 4c with $\varepsilon \propto R_{\mathrm{c}}-R_{\mathrm{i}}$. (b) Effective temporal growth rate $\varepsilon / \tau_{0}^{\text {eff }}$ of $|A|$ following small-range random initial conditions, compared to the noiseless growth rate $\varepsilon / \tau_{0}$ (dashed line).

is an important confirmation of the overall relevance of our approach in terms of noisy amplitude equations.

We have also estimated the effective values of the remaining coefficients $\tau_{0}$ and $g_{2}$. During quench experiments where the system is suddenly brought down from a fully turbulent state, we fitted the growth of the modulation to an exponential form. The corresponding growth rate provides a rough estimate of $\varepsilon / \tau_{0}^{\text {eff }}$. Our data (not shown) do show a linear variation with $\varepsilon$ which extrapolates to zero at a value which is fully consistent with the earlier determination of the threshold, so $\tau_{0}^{\text {eff }}$ can be extracted from the slope. In numerical simulations of the dynamics of Eqs. (1) following small-range random initial conditions, we measure a constant effective time $\tau_{0}^{\text {eff }}$ somewhat different from the bare value $\tau_{0}$ (Fig. 5b), but the shift is typically small compared to the experimental error bars [15].

We also took advantage of the domain walls or fronts separating $A^{-}$and $A^{+}$domains to fit the profile of $|A|$ against an exponential form $|A| \sim \exp (\lambda|z|)$. In the noiseless amplitude description, $\xi_{0}^{2} \lambda^{2} \simeq \varepsilon\left(g_{2} / g_{3}-1\right)$. Our fits are consistent with the value $g_{2} / g_{3}-1 \approx 1.2$ which according to our simulations reproduces all experimental observations, but the result is too imprecise to extract a systematic variation with $\varepsilon$. An exploration of fronts in simulations of Eqs. (1) show that the effective value of $g_{2}$ is generally smaller than its bare value [15].

In summary, we have presented two sets of remarkable observations: (i) turbulent spirals and spots are essentially the same in PCF and TCF, and correspond to a finite wavelength modulation of the turbulence intensity; (ii) all our observations are fully captured by the dynamics of coupled amplitude equations with noise. Taken together, these results suggest the possibility of a large-wavelength instability within fully turbulent shear flows. The precise origin of such an instability is at present completely unknown. The work by Hegseth [18] suggests it is related to the emergence of vortex type structures in the streamwise direction. Direct numerical simulations or flow visualization might help to clarify this. Our work also suggests new ways of looking at similar issues in other shear flows such as the Blasius boundary layer, where it has long been observed $[2,3]$ that turbulent spots can take fairly regular "V" shapes reminiscent of the inclined stripes reported here, or at the turbulent Taylor vortex flow where phase dynamics concepts have already been shown to be relevant [19].

We thank Arnaud Chiffaudel for fruitful discussions.

[1] R. P. Feynman, Lecture Notes in Physics (Addison-Wesley, Reading, MA, 1964), Vol. 2.

[2] H. W. Emmons and A. E. Bryson, J. Aerosol Sci. 18, 490 (1951).

[3] M. Gad-El-Hak, R. F. Blackwelder, and J. J. Riley, J. Fluid Mech. 110, 73 (1981).

[4] F. Alavyoon, D. S. Henningson, and P. Alfredsson, Phys. Fluids 29, 1328 (1986).

[5] D. Coles, J. Fluid Mech. 21, 385 (1965); C. Van Atta, J. Fluid Mech. 25, 495 (1966); D. Coles and C. W. Van Atta, Phys. Fluids Suppl. S120 (1967).

[6] C. D. Andereck, S. S. Liu, and H. L. Swinney, J. Fluid Mech. 164, 155 (1986).

[7] J. Hegseth, C. D. Andereck, F. Hayot, and Y. Pomeau, Phys. Rev. Lett. 62, 257 (1989).

[8] F. Daviaud, J. Hegseth, and P. Bergé, Phys. Rev. Lett. 69, 2511 (1992).

[9] O. Dauchot and F. Daviaud, Phys. Fluids 7, 335 (1995).

[10] S. Bottin and H. Chaté, Eur. Phys. J. B 6, 143 (1998).

[11] A. Prigent and O. Dauchot, Phys. Fluids 12, 10 (2000).

[12] See, e.g., M. van Hecke, C. Storm, and W. van Saarloos, Physica (Amsterdam) 134D, 1 (1999), and references therein.

[13] V. Croquette and H. Williams, Physica (Amsterdam) 37D, 300 (1989).

[14] L. Kramer and W. Pesch, Annu. Rev. Fluid Mech. 27, 515 (1995).

[15] A. Prigent, G. Grégoire, H. Chaté, and O. Dauchot (to be published).

[16] In fact, the numerically observed scaling defines an effective threshold $\left(\mathcal{A}^{2} \propto \varepsilon-\varepsilon^{*}\right)$. The shift $\varepsilon^{*}$ depends on $\alpha$, but for realistic values it remains negligible [12].

[17] In principle, $\alpha$ could be estimated from more quantitative measurements [12].

[18] J. J. Hegseth, Phys. Rev. E 54, 4915 (1996).

[19] M. Wu, C. D. Andereck, and H. R. Brand, Europhys. Lett. 19, 587 (1992). 